Material properties

\title{
Enhancing the mechanical performance of polymer based nanocomposites by plasma-modification of nanoparticles
}

\author{
Roberto Scaffaro*, Andrea Maio \\ University of Palermo, Dipartimento di Ingegneria Civile, Ambientale, Aerospaziale, dei Materiali, Viale delle Scienze, Ed. 6, 90128 Palermo, Italy
}

\section{A R T I C L E I N F O}

Article history:

Received 4 May 2012

Accepted 14 June 2012

\section{Keywords:}

Carbon nanotubes

Functionalization

Nanocomposite

Nanoclay

Nanoparticle

Plasma

\begin{abstract}
A B S T R A C T
The possibility of enhancing the mechanical performance of two different polymer-based nanocomposites using polyamide 6 (PA6) and poly[ethylene-co-(vinyl acetate)] (EVA) as matrices was investigated. The nanofillers used were, respectively, either carbon nanotubes (CNTs) or an organically modified montmorillonite (Cloisite $15 \mathrm{~A}$ ), both previously modified by plasma treatment to introduce polar moieties. The nanofillers were fully characterized by Raman spectroscopy, XPS, FT-IR and XRD, demonstrating their effective modification with oxygenated groups. The nanocomposites were prepared by melt processing in order to obtain films and fibres. The mechanical tests carried out on the nanocomposites showed a remarkable increase of the elastic modulus when plasmamodified nanoparticles were used. The improvement of wettability and dispersion of the nanofillers in the polymer matrices, as confirmed by SEM observations, can be invoked to explain this feature.
\end{abstract}

(c) 2012 Elsevier Ltd. All rights reserved.

\section{Introduction}

Polymer nanocomposites have received significant attention during the last two decades, due to the possibility of obtaining high performance materials at low filler loading. This is possible because of the higher surface-tovolume ratio of nanofillers compared to that of traditional micro-scaled fillers [1,2] However, the dispersion and the adhesion of the nanofillers in the host polymers remain the key factors for obtaining improvement of the mechanical performance [1-4]. In fact, one of the main goals in the preparation of nano-scaled composites is to prevent the nanoparticles from agglomerating in clusters or bundles. This can be achieved by introducing specific surfactants or functional groups on the nanofiller surface in order to improve compatibility with the host polymer $[2,4,5]$.

Clays and carbon nanotubes have been widely investigated and used as reinforcing agents for polymer matrices [1-8]. Clay-based nanocomposites can find applications in various areas, such as packaging and agriculture. They can

\footnotetext{
* Corresponding author. Fax: +39 (0) 916567280.

E-mail address: roberto.scaffaro@unipa.it (R. Scaffaro).
}

dramatically enhance the mechanical performance and the barrier properties at filler loadings as low as 3-5 wt\%, without significantly changing other important characteristics such as transparency or density. In order to enhance their compatibility with polymer matrices, clays are usually modified with organic compounds (e.g. quaternary ammonium salts) but, even in this case, the properties are often not significantly improved [1].

Carbon nanotubes (CNTs) have been found to be effective reinforcing agents for several polymeric materials, beyond the capability to increase their electrical and thermal conductivity [4-6]. Similarly to clays, CNTs must be modified to improve their affinity with the matrix by including specific functional moieties (e.g. carboxyls, amine, hydroxyls ...) introduced by dedicated processes [4,5,8-10]. Despite several studies reporting on plasma modification of CNTs, to the best of our knowledge, no papers about plasma carboxylation of organoclays are available in the scientific literature.

The aim of this work is the description of a rapid and solvent free method for modifying nanofillers to increase their compatibility with host polar polymers. In particular, the method aims to oxidize either carbon atoms of CNTs or those 
of the clay organic modifier, by a plasma treatment under air atmosphere, in order to introduce new polar moieties.

According to the scientific literature, clays are often used coupled with polyolefins while CNTs are preferentially used as filler in technopolymers such as polyamides, polyesters or polycarbonates [4-6,8]. For this reason, an organo-modified clay was used as filler of poly(ethyleneco-vinyl acetate) (EVA) while CNTs were added to polyamide 6 (PA6). Both the preparation and the forming steps were carried out in the melt by extrusion based processes.

\section{Experimental}

\subsection{Materials}

A commercial grade of multi-walled CNTs (Sigma Aldrich, $\mathrm{L}=5-9 \mathrm{um}, \mathrm{D}=100-170 \mathrm{~nm}$, purity $\geq 90 \%$ ) and a sample of montmorillonite modified with an alkylammonium salt (Cloisite 15A, Southern Clay) were used as nanofillers. In particular, the clay organic modifier was a dimethyl-ditallow, which displays two organophilic tails fully hydrogenated with an average composition of $65 \% C_{18}, 30 \% C_{16}$ and $5 \% C_{14}$. Both CNTs and Cloisite $15 \mathrm{~A}$ were thoroughly dried under vacuum overnight at, respectively, $80^{\circ} \mathrm{C}$ and $120^{\circ} \mathrm{C}$, prior to use.

The PA6 used for this work (Radilon S35 100 NAT inherent viscosity in sulfuric acid $3.4 \mathrm{dL} / \mathrm{g}$ ) was kindly supplied by Radicinova, Italy. In order to minimize hydrolytic scissions it was dried in a vacuum oven at $120{ }^{\circ} \mathrm{C}$ overnight prior to processing.

The EVA used was a commercial grade (Greenflex, FC45, Polimeri Europa, Italy), with a vinyl acetate content of $14 \mathrm{wt} \%$ and a melt flow index of $0.3 \mathrm{~g} / 10 \mathrm{~min}\left(\mathrm{load}=2.16 \mathrm{~kg}, \mathrm{~T}=190^{\circ} \mathrm{C}\right)$.

\subsection{Plasma modification of the nanofillers}

Both CNTs and Cloisite 15A were treated in a plasma reactor (Tucano, Gambetti, Italy) with polarized anode at radio frequency $(13.56 \mathrm{MHz})$. The plasma reactor, which has a chamber volume of about 5.5 litres, is shown schematically in Fig. 1. The process was carried out in four steps: i) reaction chamber from ambient pressure to $0.2 \mathrm{mbar}$; ii) stabilization of the inner pressure (5 s); iii) air inlet and plasma reaction (see below for further details); iv) venting to ambient pressure (about 100-150 s).

The initial pressure in the chamber was 0.2 mbar. After air inlet $(\mathrm{P}=0.3 \mathrm{mbar})$ and during plasma treatment the total pressure was 0.5 mbar. The air flow mass rate was kept at $10 \mathrm{sccm}$ by using two mass flow controllers. For the modification of CNTs, the power was set at $120 \mathrm{~W}$ and the reaction time was $500 \mathrm{~s}$, according to our previous studies [8], whereas the treatment of Cloisite $15 \mathrm{~A}$ was carried out at $50 \mathrm{~W}$ for $180 \mathrm{~s}$. CNTs underwent a stronger treatment with respect to Cloisite 15A because the cleavage of the $\mathrm{C}=\mathrm{C}$ bonds of CNTs requires higher energy than that necessary for the cleavage of the $\mathrm{C}-\mathrm{H}$ bonds of the clay organic modifier.

\subsection{Preparation of the composites}

\subsubsection{PA6/CNT}

Immediately after the plasma treatment, CNTs (2 wt\%) were premixed with solid PA6 and then fed (at $20 \mathrm{rpm}$ )

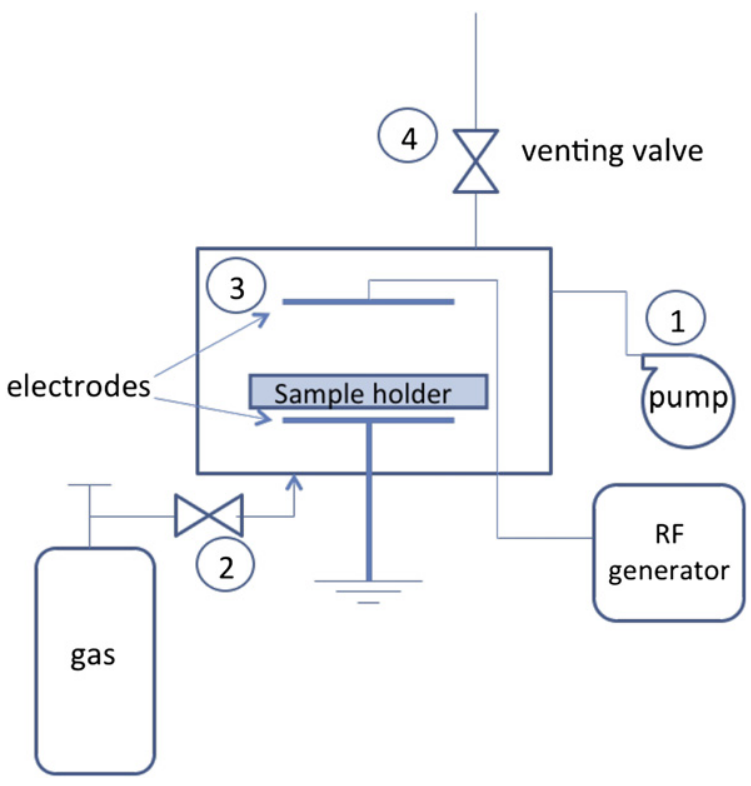

Fig. 1. Schematics of the RF-plasma reactor and of the process adopted for the modification of the nanofillers: (1) vacuuming by rotary pump; (2) gas inlet and stabilization; (3) plasma reaction; (4) venting.

to a batch mixer (Brabender) preheated at $240{ }^{\circ} \mathrm{C}$. Once the feeding stage was completed, the speed was raised to $64 \mathrm{rpm}$ and the material processed until a constant value of the mixing torque was achieved (usually after about $3 \mathrm{~min}$ ). The material was then removed from the mixer within $2 \mathrm{~min}$, any remainder being discarded.

PA6/CNTs nanocomposite fibres were prepared by using a capillary viscometer (Rheoscope 1000, CEAST, Italy) equipped with a spinning unit. The temperature was $240{ }^{\circ} \mathrm{C}$, the piston speed was $2 \mathrm{~mm} / \mathrm{min}$ and the spinning speed (collection pulley) was constantly kept at $250 \mathrm{rpm}$ to obtain fibres with a diameter of $50 \pm 3 \mu \mathrm{m}$, used for further mechanical and morphological characterization.

\subsubsection{EVA/Closite $15 A$}

Immediately after the plasma treatment, Cloisite $15 \mathrm{~A}$ (5 wt\%) was premixed with solid EVA (95 wt\%) and then fed to a single screw extruder (Brabender PLE 651, L/D $=25$, $\mathrm{D}=19$ ) equipped with a cast film die. The thermal profile adopted for the operation was $90-110-130-160{ }^{\circ} \mathrm{C}$ (from the hopper to the die), the screw speed was $64 \mathrm{rpm}$ and the drawing speed was set to $3 \mathrm{~m} / \mathrm{min}$. Under these conditions, the films had a final thickness of $100 \pm 10 \mu \mathrm{m}$.

\subsection{Nanofiller characterization}

CNTs were characterized by Raman spectroscopy (Bruker-Senterra micro-Raman equipped with a $532 \mathrm{~nm}$ diode laser excitation with $20 \mathrm{~mW}$ power). In particular, the ratio between the intensities of $D$ and $G$ bands was evaluated. This ratio, in fact, can be correlated with the degree of functionalization for the different vibration modes of carbon atoms in the presence of defects, generated during the functionalization process and associated with the 
presence of oxygen or other atoms in the nanotube structure $[5,6,8,11]$. In particular, the G-band is associated with the stretching of $\mathrm{C}=\mathrm{C}$ bonds in graphitic materials $\left(\mathrm{sp}^{2}\right.$ hybridization), while the D-band is associated with phonons close to the boundary of the Brillouin zone, usually detectable by Raman spectroscopy for disordered crystals (defects in $\mathrm{sp}^{2}$ carbons).

X-ray photoelectron spectroscopy (XPS) was carried out by a Perkin-Elmer PHI 5600 to determine the oxygen content on the surface of the CNTs before and after the functionalization. More details and results can be found elsewhere [8]. Cloisite 15A nanoparticles have been characterized by FTIR spectroscopy (Perkin Elmer Spectrum 400 ) by dispersing them in $\mathrm{KBr}$ with a ratio Closite: $\mathrm{KBr}=1: 20$ by weight. The morphology was investigated by X-ray diffraction (XRD) (Italiastructures APD 2000, Italy). To improve the quality of the signal, XRD tests were carried out on specimens ( $2 \mathrm{~mm}$ thick) compacted under pressure (100 bar, $\mathrm{t}=5 \mathrm{~min}, \mathrm{~T}=20^{\circ} \mathrm{C}$ ).

\subsection{Nanocomposites}

The tensile properties of PA6/CNTs fibers and EVA/ Cloisite 15A films were measured using a dynamometer, Instron 3365 (UK). The crosshead speed was $1 \mathrm{~mm} / \mathrm{min}$ for the first two minutes and $100 \mathrm{~mm} / \mathrm{min}$ thereafter. The grip distance was $30 \mathrm{~mm}$ and the results reported are the average of at least 15 replicates.

The morphological analysis of the composites was evaluated by scanning electron microscopy (SEM FEI Quanta 200) on samples fractured under nitrogen and sputter-coated with a thin layer of gold to avoid electrostatic charging under the electron beam.

\section{Results and discussion}

\subsection{Nanoparticles modification}

Plasma functionalization is supposed to provide both CNTs and Cloisite 15A with oxygenated moieties (carboxyls, carbonyls, hydroxyls, ...). CNT modifications can be easily followed by Raman and XPS spectroscopy. The Raman spectra of neat and functionalized CNTs (fCNTs) are reported in Fig. 2a in which it is possible to identify the D-band and the G-band, respectively centered at 1339 and $1569 \mathrm{~cm}^{-1}$. The ID/IG ratio of neat CNTs is 0.08 and it increases to 0.14 after plasma treatment. The conversion of the carbons of the graphenic outer walls from $\mathrm{sp}^{2}$ to $\mathrm{sp}^{3}$ once they bind with oxygen can be reasonably invoked to explain this result. This hypothesis is corroborated by XPS. Fig. 2b reports the 01s HR-XPS for CNTs and fCNTs. Neat CNTs present small amounts of oxygen, probably due to the manipulation/manufacturing of the commercial nanotubes used in this work. After plasma treatment, the amount of oxygen is almost doubled, thus confirming the formation of new oxygenated moieties. The plasma treatment had similar effects on Cloisite 15A. Fig. 3 presents the FTIR spectra of Cloisite 15A, before and after plasma treatment, with a close-up inset of the carbonyl region. Table 1 reports the assignments of the main bands according to the scientific literature [12,13].

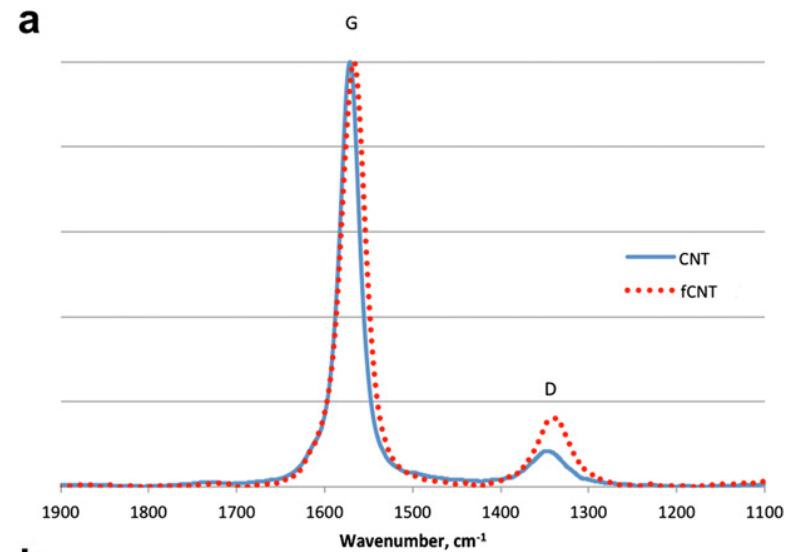

b

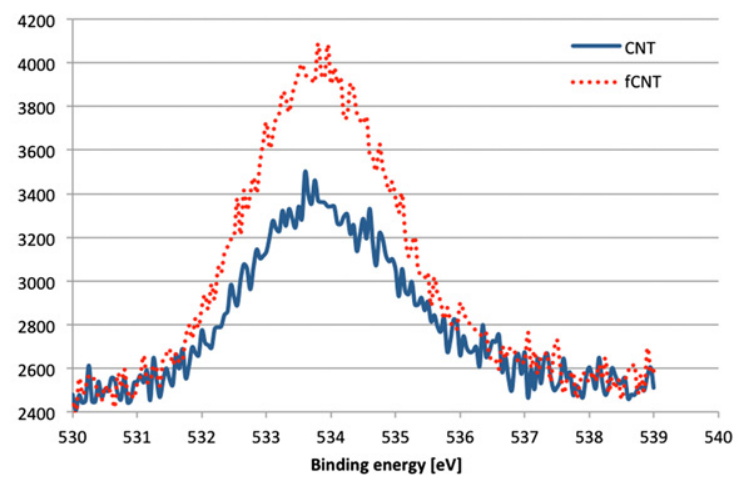

Fig. 2. Raman spectra focused on D-band and G-band (a) and 01s signal recorded by XPS spectra (b) for CNTs (solid line) and fCNTs (dotted line).

It is evident that, after the functionalization, the carbonyl region is modified. The band at $1640 \mathrm{~cm}^{-1}$, related to the stretching vibrations of the quaternary ammonium salts, is reduced and, contextually, the band at $1718 \mathrm{~cm}^{-1}$, assigned to the carboxyl stretching, is more intense. Therefore, it can be qualitatively hypothesized that, to some extent, carbons on the alkylic tail of the clay organic modifier converted to carboxyls. In addition, the spectrum is significantly different in the region around $1033-1040 \mathrm{~cm}^{-1}$, i.e. the region of the vibration of $\mathrm{Si}-\mathrm{O}$ and $\mathrm{Si}-\mathrm{O}-\mathrm{Si}$ bonds. In the plasma-treated sample these bands are drastically reduced. This feature suggests a significant change in the clay lamellar organization that influences the resonance vibration energy and, consequently, the spectrum. This is corroborated by XRD analysis, Fig. 4. For both neat and treated Cloisite 15A the characteristic peak at about $2.8^{\circ}$ is clearly visible, corresponding to an interlayer distance of $3.15 \mathrm{~nm}$. However, the treated sample displays a weaker and broader band that can be attributed to increased disorder of the lamellae (disruption after plasma treatment).

\subsection{Nanocomposites characterization}

\subsubsection{PA6/CNTS}

The mixing torque as a function of time for PA6 based materials is reported in Fig. 5. This property is related to the 


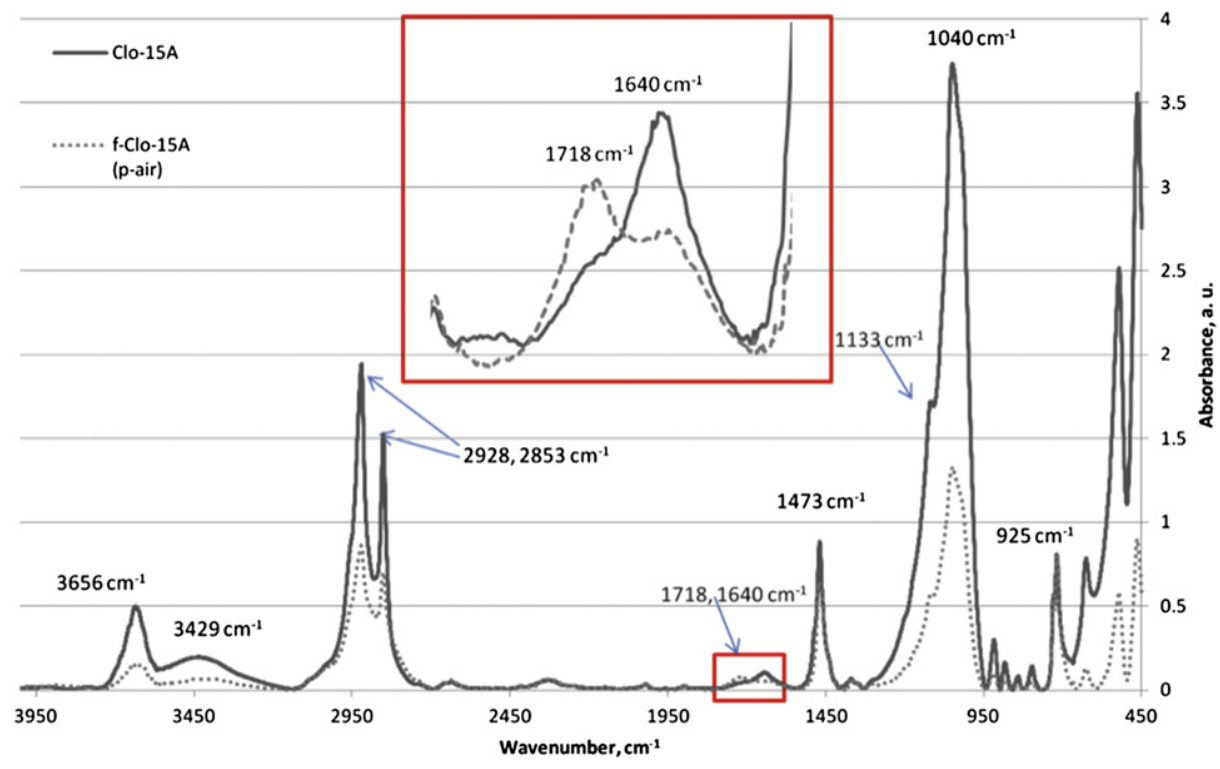

Fig. 3. FT-IR spectra of Cloisite $15 \mathrm{~A}$ (solid line) and air-plasma modified Cloisite $15 \mathrm{~A}$ (dotted line).

viscosity of the material during processing and it can give information about the rheological behaviour of the materials.

Adding CNTs to PA6 causes a remarkable increase of the mixing torque. This result is expected, as the fillers reduce the macromolecules mobility compared with that of the neat polymer, thus causing an increase of viscosity and, therefore, of the mixing torque. Adding CNTs causes an increase of torque from about $7.5 \mathrm{Nm}$ of PA6 to about $8.5 \mathrm{Nm}$ of PA6/CNTs. The increment is even higher (about $9 \mathrm{Nm}$ ) when fCNTs are added. This feature can be explained considering that the oxygenated groups formed on fCNTs due to the treatment are responsible for improved interfacial adhesion that causes an increase of viscosity. The morphological analysis, Fig. 6a-b carried out on PA6/CNTs, Fig. 6a, and PA6/fCNTs, Fig. 6b, provides evidence that neat CNTs tend to agglomerate and to be pulled out the matrix, thus showing poor adhesion and poor dispersion in the PA6 matrix. When fCNTs are used, both the adhesion and especially the dispersion improve.

In order to induce the orientation of the PA6 macromolecules and of the CNTs along a preferential direction, PA6 based materials were spun to obtain fibres. The related

Table 1

Band assignments of the FT-IR spectra reported in Fig. 3.

\begin{tabular}{ll}
\hline Band $\left(\mathrm{cm}^{-1}\right)$ & Assignments \\
\hline 3656 & OH stretching from silicate \\
3429 & Adsorbed water \\
2928,2853 & C-H stretching (aliphatic) \\
1718 & Carboxyl stretching \\
1640 & Quaternary ammonium salts \\
1473 & Ethylene bending \\
1133 & Si-O Stretching \\
1040 & Si-O-Si Stretching \\
925 & Al-OH stretching \\
\hline
\end{tabular}

tensile properties are reported in Table 2. Adding CNTs causes an increase of the elastic modulus with respect to pure PA6, probably due to the stiffening of the matrix

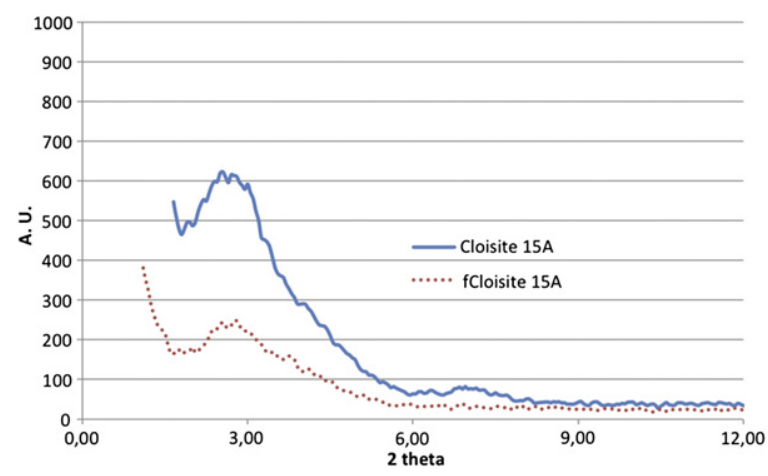

Fig. 4. XRD patterns of neat and modified Cloisite 15A.

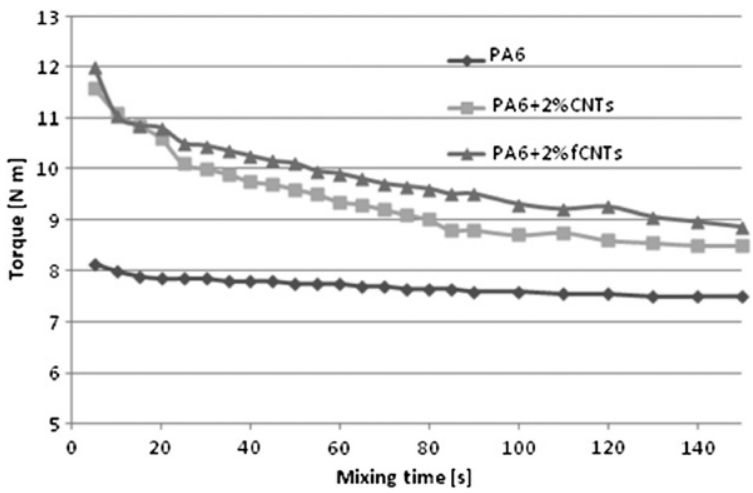

Fig. 5. Torque as a function of mixing time during the melt processing. 


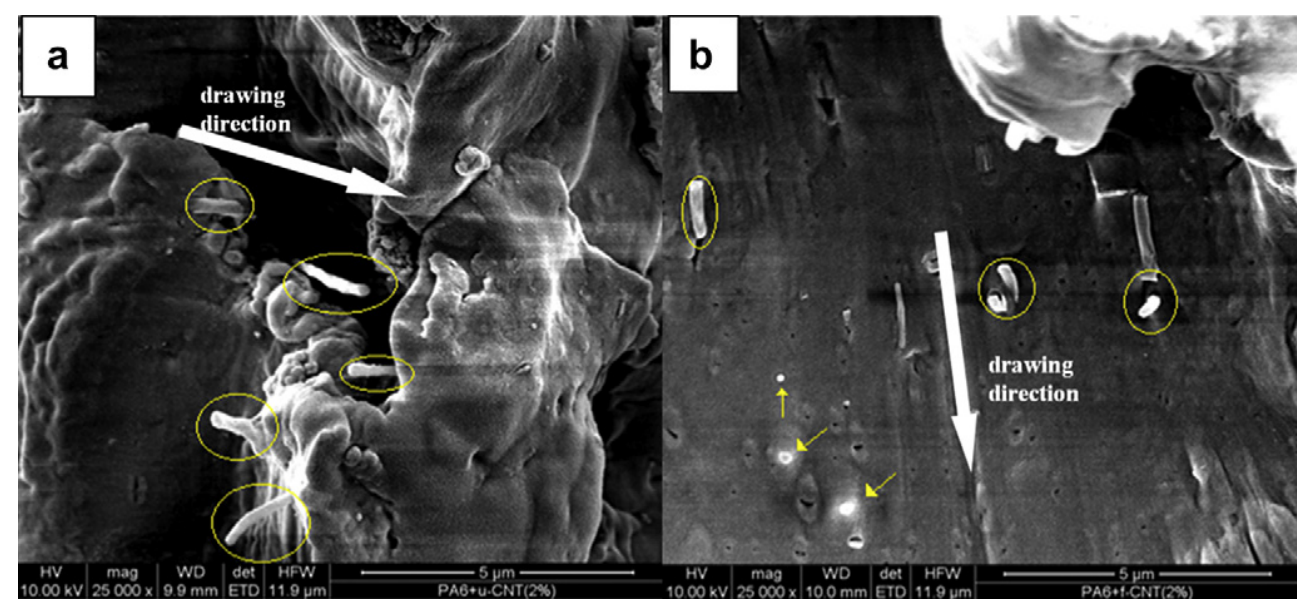

Fig. 6. SEM micrographs of fibers containing $2 \%$ of untreated (a) and modified (b) CNTs.

Table 2

Mechanical properties of neat PA6 and nanocomposites filled with untreated and modified CNTs.

\begin{tabular}{llll}
\hline Sample & E $(\mathrm{MPa})$ & TS $(\mathrm{MPa})$ & EB $(\%)$ \\
\hline PA6 & $1831 \pm 67$ & $36.2 \pm 8.7$ & $332 \pm 37$ \\
PA6/CNTs & $2441 \pm 89$ & $26.1 \pm 3.8$ & $248 \pm 27$ \\
PA6/fCNTs & $3791 \pm 45$ & $33.0 \pm 4.1$ & $271 \pm 9$ \\
\hline
\end{tabular}

induced by the presence of the nanofillers, as widely observed before [4-6,8,10,14-16]. The modulus is even higher when fCNTs are used instead. In this case, the modulus is about the double that observed for the PA6/CNT system and about three times compared with pure PA6. Again, these results can be interpreted considering that fCNTs bearing polar groups on their surface are likely more adherent and well dispersed in the PA6 matrix. Moreover, the orientation of the CNTs under the elongational flow can cause the increase of the elastic modulus here observed.

These hypotheses find confirmation in the morphological analysis, Fig. 6a-b. In both PA6/CNTs, Fig. 6a, and PA6/ fCNTs, Fig. 6b, a preferential orientation of the nanotubes along the drawing direction (evidenced by the arrows can be seen).
As regards the properties at break, Table 2, both tensile stress and elongation at break decrease by adding CNTs to PA6. As is well known, this is a direct consequence of the structure stiffening after adding the nanofiller to the matrix. When fCNTs are used, however, both properties increase and become similar to those of neat PA6. This is probably due to the already mentioned better adhesion and dispersion of fCNTs that induce better stress transfer from the matrix to the filler and, consequently, increased deformability of the material.

\subsubsection{EVA/Cloisite $15 A$}

Similarly to the PA6/CNTs systems, for EVA/Cloisite 15A also it is possible to observe an improvement of the elastic modulus of the composites. Table 3 reports the tensile properties of EVA with neat and modified Cloisite 15A

\section{Table 3}

Mechanical properties of neat EVA and nanocomposites filled with untreated and functionalized Cloisite 15A.

\begin{tabular}{lllr}
\hline Sample & E (MPa) & TS (MPa) & \multicolumn{1}{c}{ EB (\%) } \\
\hline EVA & $36.6 \pm 1.8$ & $25.3 \pm 1.5$ & $1155 \pm 70$ \\
EVA/Cloisite 15A & $43.5 \pm 2$ & $17.3 \pm 1$ & $804 \pm 40$ \\
EVA/fCloisite 15A & $52.2 \pm 2.2$ & $20.5 \pm 1.1$ & $1003 \pm 60$ \\
\hline
\end{tabular}

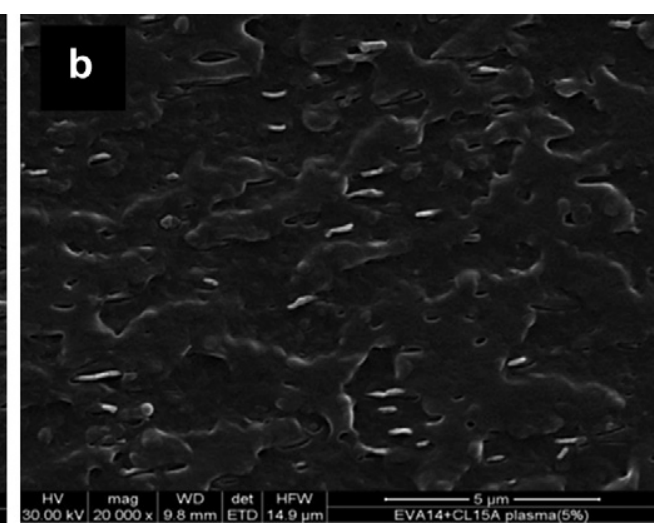

Fig. 7. SEM micrographs of EVA-based films filled with untreated (a) and modified (b) Cloisite 15A. 
together with those of pure EVA, for comparison. The modulus increases when Cloisite $15 \mathrm{~A}$ is added but the highest values are observed in the composites containing fCloisite 15A. Again, an improved interaction between the matrix and the filler can be invoked to explain the results. Fig. 7 shows the SEM micrographs of EVA with Cloisite 15A, Fig. 7a, and with plasma modified Cloisite 15A, Fig. 7b. The dispersion of the nanofiller in the system containing fCloisite $15 \mathrm{~A}$ is dramatically better than that observed when neat Cloisite 15A is added. An image analysis elaboration of different SEM micrographs showed that the average amount of particles passed from 0.46 particles $/ \mu \mathrm{m}^{2}$ of neat Cloisite 15 A to 0.86 particles $/ \mu \mathrm{m}^{2}$ of fCloisite $15 \mathrm{~A}$. This is probably due to the aforementioned destructuring of the clay tactoids confirmed by FTIR and XRD analysis. The properties at break, Table 3, follow the same trend observed for PA6/CNTs systems. Adding neat Cloisite 15A causes a decrease of both elongation at break and tensile stress, but when fCloisite $15 \mathrm{~A}$ is used they both increase, getting closer to the values displayed by the pure matrix. This feature is further indirect evidence of the enhancement of adhesion and dispersion already mentioned above.

\section{Conclusions}

In this work, two nanofillers, namely carbon nanotubes (CNTs) and an organo-clay (Cloisite 15A), were modified by air plasma in order to improve their affinity with ad hoc dedicated matrices: polyamide 6 (PA6) for CNTs and poly(ethylene-co-vinyl acetate) (EVA) for the organoclay.

The nanofiller modification has been confirmed by spectroscopic analyses and the results indicated that plasma treatment leads to the oxidation of graphenic carbons in CNTs and aliphatic carbons of the organic modifier in Cloisite15A.
When modified nanofillers are added to the polymer matrices, both the adhesion and the dispersion are improved and, as a consequence, the mechanical performance is enhanced. The elastic modulus increases both for PA6-fCNT (about triple if compared to neat PA6) and for EVA/fCloisite15A ( $+45 \%$ with respect to neat EVA). The properties at break, that typically decrease when fillers are added, are comparable with those of the neat matrix when modified nanofillers are used.

\section{References}

[1] S.S. Ray, M. Okamoto, Prog. Polym. Sci. 28 (2003) 1539-1641.

[2] M. Alexandre, P. Dubois, Mater. Sci. Eng. R 28 (2000) 1-63.

[3] J.-H. Chang, Y. Uk-An, G.S. Sur, J. Polym. Sci. Polym. Phys. 41 (1) (2003) 94-103.

[4] Z. Spitalsky, D. Tasis, K. Papagelis, C. Galiotis, Prog. Polym. Sci. 35 (3) (2010) 357-401.

[5] N. Grossiord, H.E. Miltner, J. Loos, J. Meuldijk, B.V. Mele, Koning. Chem. Mater. 19 (15) (2007) 3787-3792.

[6] J.K.W. Sandler, J.E. Kirk, I.A. Kinloch, M.S.P. Shaffer, A.H. Windle, Polymer 44 (19) (2003) 5893-5899.

[7] R. Scaffaro, L. Botta, M. Ceraulo, F.P. La Mantia, J. Appl. Polym. Sci. 122 (1) (2011) 384-392.

[8] R. Scaffaro, A. Maio, S.P. Agnello, A. Glisenti, Plasma Process, Polym. 9 (5) (2012) 503-512.

[9] V. Georgakilas, K. Kordatos, M. Prato, D.M. Guldi, M. Holzingger, A. Hirsch, J. Am. Chem. Soc. 124 (5) (2002) 760-761.

[10] J.Y. Yook, J. Jun, S. Kwak, Appl. Surf. Sci. 256 (23) (2010) 6941-6944.

[11] M.S. Dresselhaus, A. Jorio, M. Hofmann, G. Dresselhaus, R. Saito, Nano Lett. 10 (3) (2010) 751-758.

[12] R. Scaffaro, L. Botta, M.C. Mistretta, F.P. La Mantia, Polym. Degr. Stab. 95 (12) (2010) 2547-2554.

[13] R. Scaffaro, M.C. Mistretta, F.P. La Mantia, Polym. Degr. Stab. 93 (7) (2008) 1267-1274

[14] G.X. Chen, H.S. Kim, B.H. Park, J.S. Yoon, Polymer 47 (13) (2006) 4760-4767.

[15] M. Morcom, K. Atkinson, G.P. Simon, Polymer 51 (15) (2010) 35403550.

[16] O. Meincke, D. Kaempfer, H. Weickmann, C. Friedrich, M. Vathauer, H. Warth, Polymer 45 (3) (2004) 739-748. 